\title{
Image-Enhanced Endoscopy and Its Corresponding Histopathology in the Stomach
}

\author{
Hisashi Doyama ${ }^{1}$, Hiroyoshi Nakanishi', and Kenshi $\mathrm{Yao}^{2}$ \\ ${ }^{1}$ Department of Gastroenterology, Ishikawa Prefectural Central Hospital, Kanazawa, and ${ }^{2}$ Department of Endoscopy, Fukuoka University \\ Chikushi Hospital, Chikushino, Japan
}

\author{
Article Info \\ Received November 18, 2019 \\ Revised January 24, 2020 \\ Accepted January 25, 2020 \\ Published online March 25, 2020

\section{Corresponding Author} \\ Kenshi Yao \\ ORCID https://orcid.org/0000-0003-0863-3649 \\ E-mail yao@fukuoka-u.ac.jp
}

In recent years, the technological innovation and progress of endoscopic equipment have been remarkable, and various endoscopic observation techniques have been developed. Among them, representative techniques are magnified observation and narrow-band imaging. Magnifying endoscopy with narrow-band imaging (M-NBI) can visualize superficial microanatomies in the stomach. The normal morphology of the microanatomy visualized using M-NBI differs according to the part of the stomach. The vessel plus surface (VS) classification system has been developed as a diagnostic criterion for early gastric cancer using $\mathrm{M}-\mathrm{NBI}$, and its usefulness has been proven. Based on the VS classification system, a magnifying endoscopy simple diagnostic algorithm for early gastric cancer (MESDA-G), a simplified algorithm used for early gastric cancer diagnosis, was created. We aimed to describe the anatomic structure of the stomach that can be viewed using M-NBI and outline the principles and clinical application of the VS classification system and MESDA-G. (Gut Liver 2021;15:329-337)

Key Words: Early gastric cancer; Endoscopy; Narrow band imaging; Magnifying endoscopy

\section{INTRODUCTION}

Various categories of image-enhanced endoscopy are applied clinically. ${ }^{1}$ By using image-enhanced endoscopy in combination with a magnifying endoscope, minute anatomical structures, such as microvascular architecture (V) and microsurface structure (S), can be clearly observed. ${ }^{2,3}$ In this paper, we aimed to describe the anatomic structure of the stomach that can be visualized using a magnifying endoscopy with narrow-band imaging (M-NBI), and its diagnostic system for gastric cancer, whose usefulness has been proven by strong evidence in the diagnosis of early gastric cancer. $^{4-9}$

\section{OBSERVATION WITH M-NBI IN THE STOMACH}

Using a black soft hood is necessary to obtain an optimal magnified endoscopic image. ${ }^{6}$ The hood ensures the distance between the mucosal surface and the tip of the endoscope to be maintained at about $2 \mathrm{~mm}$, whereby fine endoscopic image of optimum maximum magnification can be obtained.

\section{MICROANATOMIES IN THE STOMACH AS VISUALIZED USING M-NBI}

The microanatomical components visualized using $\mathrm{M}-\mathrm{NBI}$ are $\mathrm{V}$ and $\mathrm{S}^{2,3}$ Using correct anatomical terms is important to analyze these M-NBI findings (Table 1). As for the $\mathrm{V}$, the subepithelial capillary network (SECN), the

Table 1. Microanatomies Visualized by Magnifying Endoscopy with Narrow-Band Imaging in the Stomach

\begin{tabular}{ll}
\hline \multicolumn{1}{c}{ Microvascular pattern: $V$} & \multicolumn{1}{c}{ Microsurface pattern: S } \\
\hline 1. Subepithelial capillary network & 1. Marginal crypt epithelium \\
2. Collecting venule & 2. Crypt opening \\
3. Microvessel & 3. Intervening part \\
& 4. Light blue crest \\
& 5. White opaque substance \\
\hline
\end{tabular}



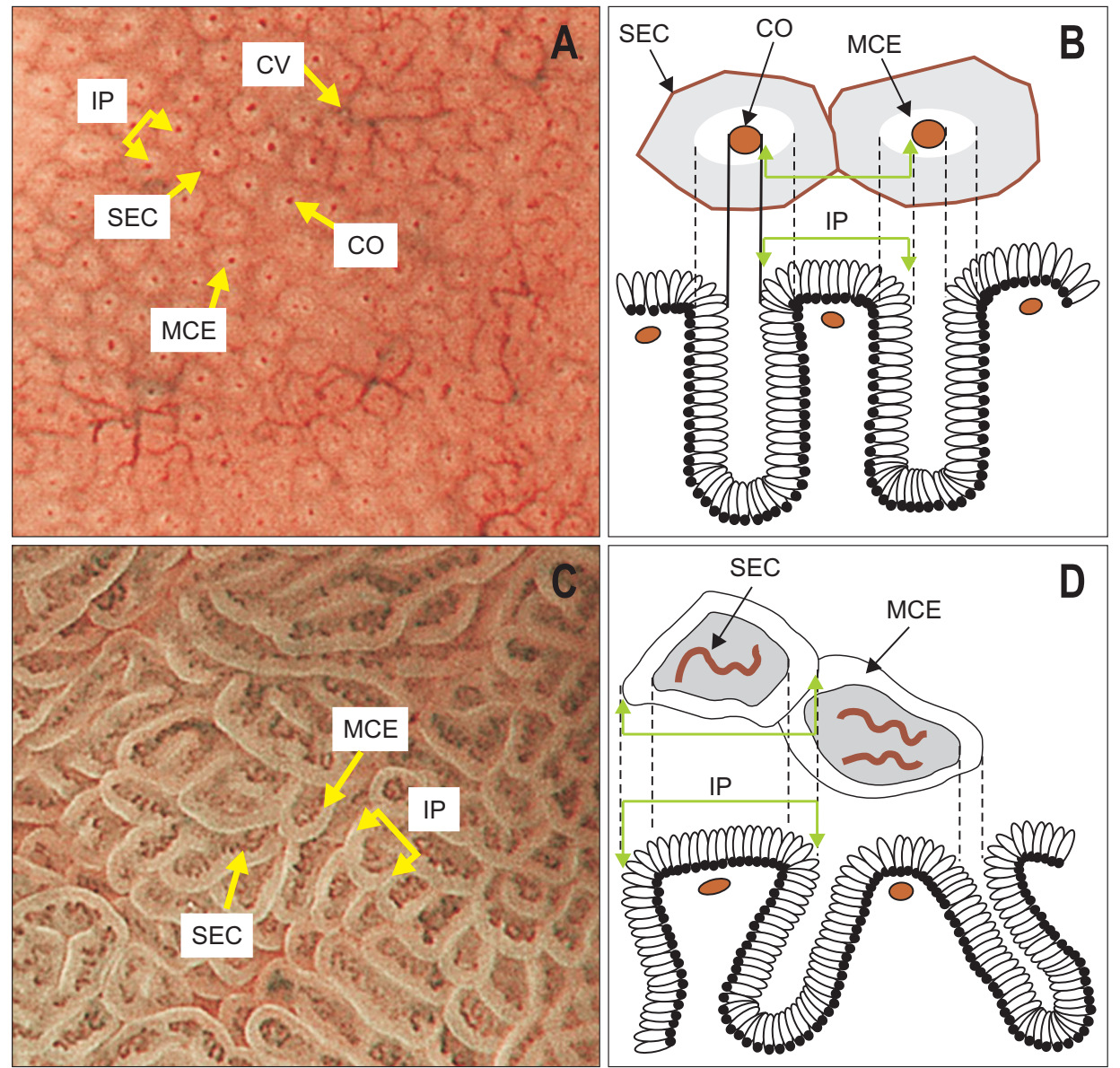

Fig. 1. Magnifying endoscopy with narrow-band imaging (M-NBI) findings of normal gastric mucosa and corresponding histological findings. (A) Endoscopic image of normal gastric fundic gland mucosa visualized using M-NBI. A dark brown oval-shaped crypt opening (CO) is lined with white semitransparent oval marginal crypt epithelium (MCE) surrounded by subepithelial capillaries (SECs). These capillaries form a regular honeycomb-like subepithelial capillary network pattern and are drained into a cyan-colored collecting venule (CV). The area between crypts is referred to as the intervening part (IP). (B) Relationship between M-NBI findings (upper panel) and histological findings (lower panel) in the superficial portion of the normal gastric fundic gland mucosa. (C) Endoscopic image of normal gastric pyloric gland mucosa visualized using M-NBI. The IP is lined with curved or polygonal white semitransparent MCE. Coil-shaped SECs are observed underneath the surface epithelium of the IP. (D) Relationship between M-NBI findings (upper panel) and histological findings (lower panel) of the superficial portion of the normal gastric pyloric gland mucosa.
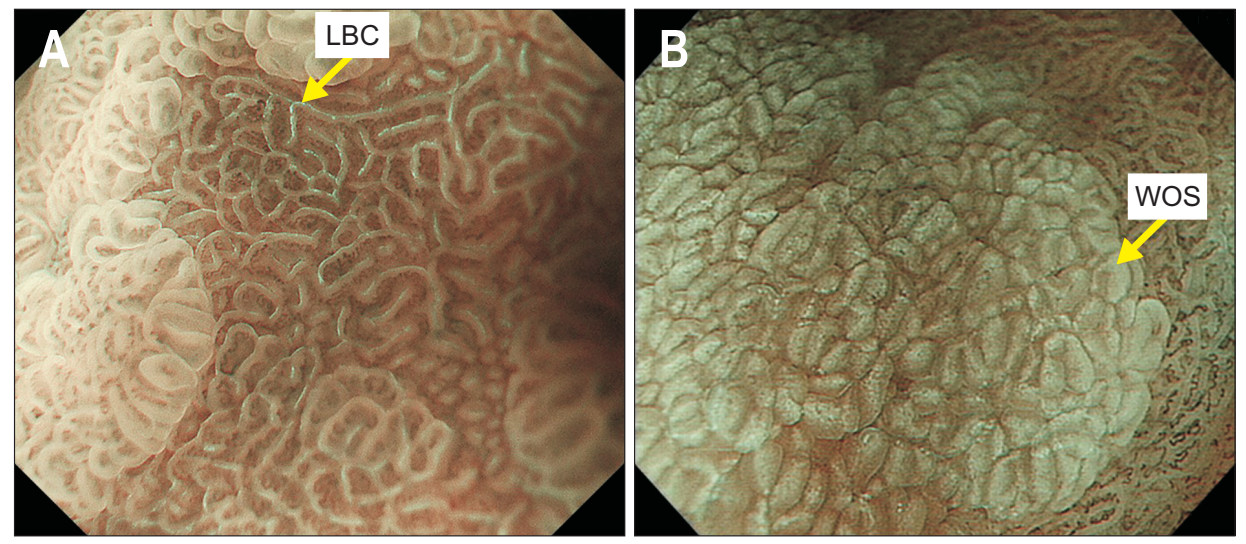

Fig. 2. Endoscopic image of the light blue crest (LBC) and white opaque substance (WOS) visualized using magnifying endoscopy with narrow-band imaging. (A) The LBC appears as blue-white lines on the epithelial border (yellow arrow). (B) Lipid micro-droplets, visualized as a WOS lyellow arrow), accumulated within the epithelium cause the projected light to reflect and strongly backward scatter and are. 
collecting venule (CV), and the microvessel (MV) are visualized. ${ }^{2,3}$ The MV is a term used for the vessels that appear in pathological mucosa. As for the $\mathrm{S}$, the marginal crypt epithelium (MCE), the crypt opening (CO), and the intervening part (IP) between crypts are the major anatomical components. ${ }^{2,3}$ These morphologies differ in gastric fundic gland mucosa (Fig. 1A and B) and gastric pyloric gland mucosa (Fig. 1C and D).

In some pathological mucosa, a light blue crest $(\mathrm{LBC})^{10}$ or a white opaque substance (WOS) ${ }^{11}$ is visualized and can be a marker for S (Fig. 2). Uedo et al. ${ }^{10}$ observed LBC only with NBI, LBC is defined as a fine, blue-white line seen at the epithelial border. Histologically, it is a marker of brush border on the epithelial surface of intestinal metaplasia. WOS is a M-NBI finding reported by Yao et al. ${ }^{11,12}$ It is a white substance in the superficial area of gastric neoplasia with intestinal phenotype or intestinal metaplasia that obscures the subepithelial V. WOS is lipid micro-droplet that is densely accumulated within the epithelium or beneath the mucosal epithelium. ${ }^{12,13}$ The morphologic differences in WOS are used as an optical microsurface marker for discriminating adenomas from adenocarcinomas. ${ }^{11}$

\section{Normal fundic gland mucosa}

The normal fundic gland mucosa indicates a corpus mucosa without any pathological changes, such as inflammation and atrophy caused by Helicobacter pylori infection. The capillary branched from submucosal arterioles penetrates the muscularis mucosa and distributes toward the mucosal surface surrounding the glandular duct while repeating anastomosis with each other in the mucosa. ${ }^{14}$ These mucosal capillaries form a regular honeycomb-like SECN in the subepithelial region and is drained into the veins in the subepithelial layer via the CVs. ${ }^{14,15}$

The $\mathrm{V}$ of the normal fundic gland mucosa visualized using M-NBI is the capillary and CV (Fig. $1 \mathrm{~A}$ and B). The morphology of the capillary is a dark brown polygonal closed loop. These capillaries are anastomosed together to form a regular honeycomb-like SECN pattern. SECN is drained into a cyan-colored $\mathrm{CV}^{2,3}$ Starfish-like CVs are regularly arranged in the normal fundic gland mucosa without $H$. pylori infection. ${ }^{16}$ As for the $\mathrm{S}$, a dark brown oval CO is surrounded by a white semi-transparent MCE (Fig. 1A and B). The area between crypts is the IP. ${ }^{2,3}$

\section{Normal pyloric gland mucosa}

The normal pyloric gland mucosa indicates a pyloric mucosa without any pathological changes, such as inflammation and intestinal metaplasia caused by $H$. pylori infection. Similar to the fundic gland mucosa, the capillary branched from the submucosal arterioles penetrates through the muscularis mucosa and is distributed in the lamina propria, whereas some arterioles penetrate the muscularis mucosa and branch the capillary within the lamina propria. The capillary is less dense than the fundic gland mucosa. These capillaries are distributed under the surface epithelium of IP while repeating anastomosis. Since these mucosal capillaries, in contrast to the fundic gland mucosa, perfuse the CV located at the deeper part of the mucosa. ${ }^{14,15}$

The $\mathrm{V}$ of the normal pyloric gland mucosa visualized using M-NBI is often only capillary, and few CVs are visualized (Fig. 1C and D). As mentioned above, this is because $\mathrm{CV}$ is located at the deeper part of the mucosa. The morphology of the capillary is a dark brown coil-shaped open loop. As for the S, polygonal or curved MCE is visualized (Fig. 1C and D). The part bordered by MCE is IP. The dark brown $\mathrm{CO}$ is rarely visualized. ${ }^{2,3}$
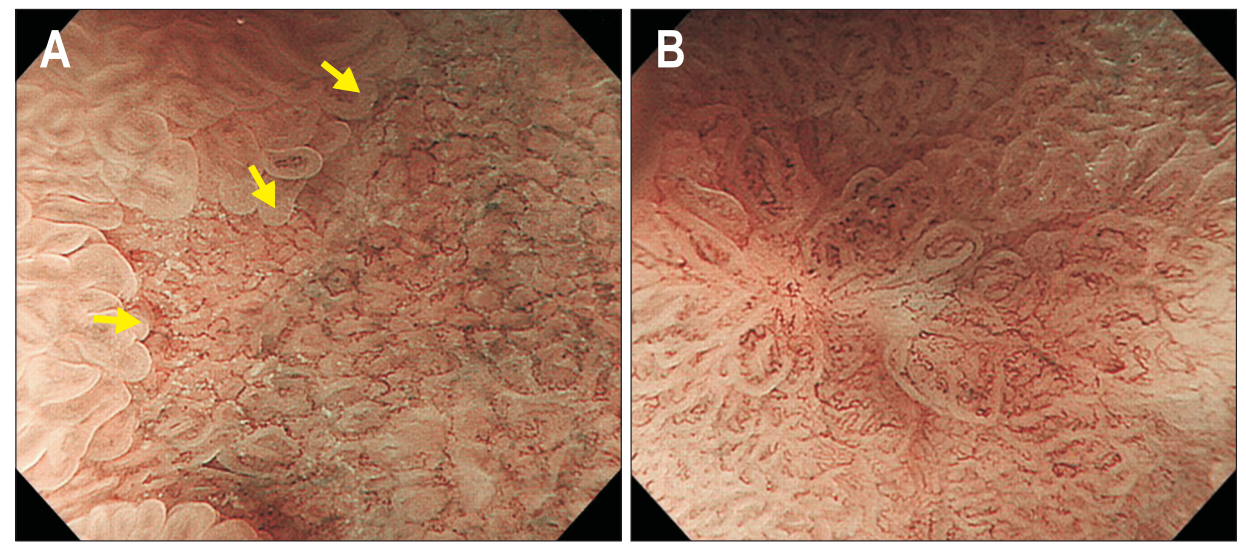

Fig. 3. Representative magnifying endoscopy with narrow-band imaging (M-NBI) findings of a demarcation line (DL). M-NBI findings (A) with a DL (yellow arrows) and (B) without a DL. 


\section{VESSEL PLUS SURFACE CLASSIFICATION SYSTEM FOR THE DIAGNOSIS OF EGC}

The vessel plus surface (VS) classification system proposed by Yao et al. ${ }^{4,6}$ has proven to be very useful in distinguishing between early gastric cancer (EGC) and non-can$\mathrm{cer}^{5,7-9}$ and delineating the margins of EGC..$^{17,18}$ According to the VS classification system, the characteristics of the MNBI findings in gastric cancer are the presence of a clear demarcation line (DL) between the noncancerous and the cancerous mucosa, and the presence of an irregular microvascular (MV) pattern and/or irregular microsurface (MS) pattern inside the DL. ${ }^{6}$

Accordingly, criteria were set for making a diagnosis of EGC with M-NBI as follows: (1) an irregular MV pattern with a DL; and/or (2) an irregular MS pattern with a DL.

$\mathrm{DL}$ is a border between the lesion and non-lesion areas and can be identified as an abrupt change of MV and/or MS patterns (Fig. 3). In principle, MV and MS patterns are analyzed separately. The three categories of MV and MS patterns are regular, irregular, and absent.
In the regular MV pattern (Fig. 4A), each mucosal capillary has a shape that is closed-looped (polygonal) or openlooped with homogeneous morphology. Moreover, its distribution and arrangement are symmetrical and regular, respectively. In the irregular MV pattern (Fig. 4B), each mucosal capillary has a different shape, such as closedlooped (polygonal), open-looped, tortuous, or branched with heterogeneous morphology. Its distribution is asymmetric, and the arrangement is irregular. Cancer-specific morphology of irregular MV is described as heterogeneity in shape. ${ }^{19}$ The MV pattern is classified as absent when it is not sufficiently visible due to the presence of WOS (Fig. 4C). In such lesions, morphological analysis of the WOS could be an alternative marker of MS pattern. ${ }^{6,11,12}$

In the regular MS pattern (Fig. 5A), each MCE is a uniform linear, curved, oval, or circular structure with homogeneous morphology. Its distribution and arrangement are symmetrical and regular, respectively. In the irregular MS pattern (Fig. 5B), each MCE is an irregular linear, curved, oval, circular, or villous structure with heterogeneous morphology. Its distribution is asymmetric, and the ar-
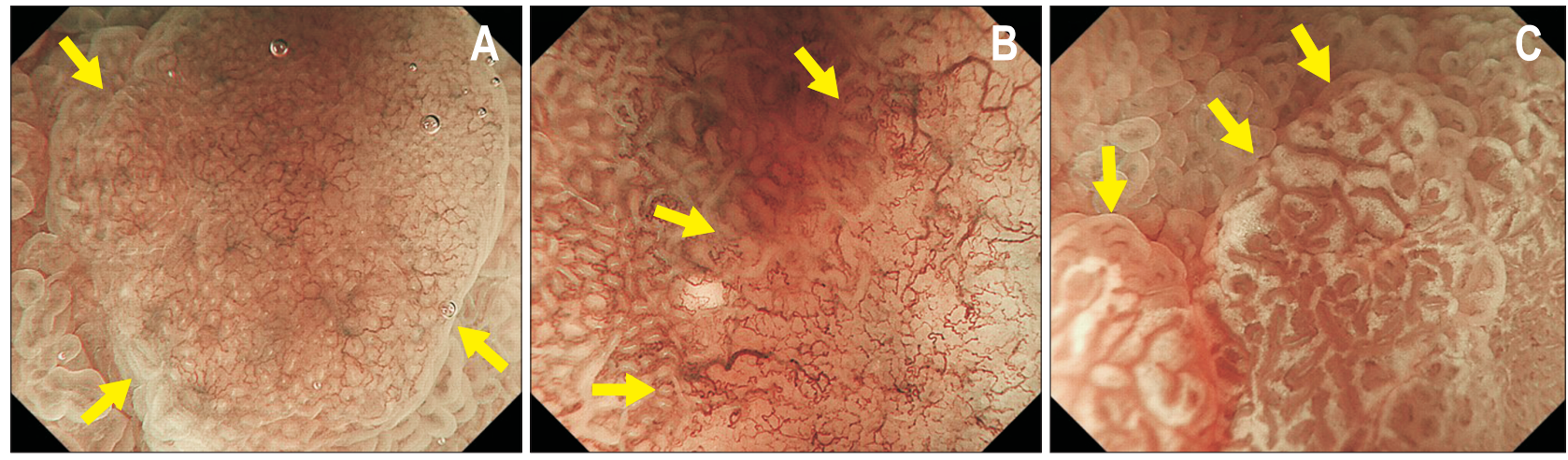

Fig. 4. Representative magnifying endoscopy with narrow-band imaging findings of a microvascular (MV) pattern. (A) Regular MV pattern. (B) Irregular MV pattern. (C) Absent MV pattern. The MV architecture cannot be observed due to the white opaque substance. Yellow arrows indicate a demarcation line.
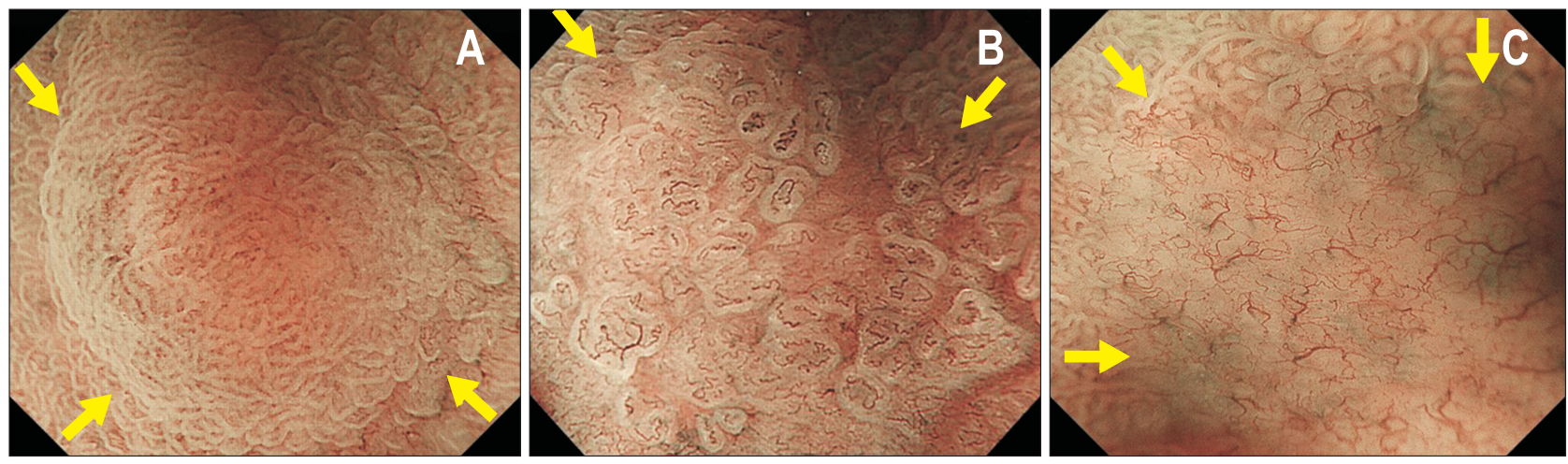

Fig. 5. Representative magnifying endoscopy with narrow-band imaging findings of a microsurface (MS) pattern. (A) Regular MS pattern. (B) Irregular MS pattern. (C) Absent MS pattern. Yellow arrows indicate a demarcation line. 
rangement is irregular. If neither the MCE nor the WOS is visible using M-NBI, the MS pattern is classified as absent (Fig. 5C).

Although the VS classification system could be expected to provide high accuracy (97\%), high positive predictive value (79\%), and high negative predictive value (99\%) for diagnosis of EGC, ${ }^{7}$ pale superficial depressed/flat lesions observed using conventional white light imaging (CWLI) may be limitations of M-NBI. ${ }^{9}$ Such pale superficial depressed lesion observed by C-WLI is characteristic of a signet-ring cell or undifferentiated-type carcinoma. These cancers may be visualized as a regular MV pattern and a regular MS pattern without a DL in M-NBI, as they are sparsely infiltrated under surface epithelium without destroying the mucosal epithelium or subepithelial capillaries.

\section{OTHER M-NBI FINDINGS USEFUL FOR DIAGNOSIS OF EGC}

The usefulness of M-NBI has been reported since it became available in clinical practice. The clinical application and usefulness of M-NBI for the diagnosis of EGC are described below.

\section{Vessels within an epithelial circle pattern}

A vessels within an epithelial circle (VEC) pattern is the presence of vessels within the circular IP surrounded by circular MCE (Fig. 6A). The circular IP surrounded by circular MCE visualized using M-NBI corresponds histologically to the papillary IP (Fig. 6B) ${ }^{20}$ While the observation of a VEC pattern itself does not indicate a carcinoma, the VEC pattern observed in the cancer area is a marker for papillary structure of adenocarcinoma. In addition, onefourth of VEC-positive EGCs have coexisting undifferentiated-type carcinoma and submucosal invasion. Therefore,
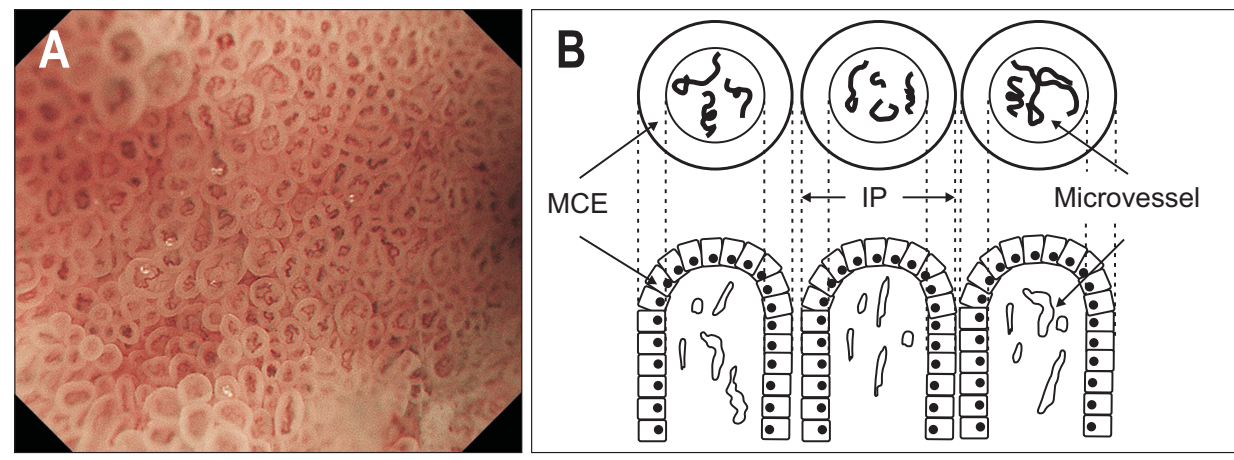

Fig. 6. Endoscopic image of vessels with an epithelial circle (VEC) pattern and a corresponding histopathological structure. (A) Magnifying endoscopy with narrow-band imaging (M-NBI) findings of a VEC pattern. The microvessels are present beneath the circular intervening part (IP) between crypts lined by circular marginal crypt epithelium (MCE). (B) Relationship between the VEC pattern as visualized using M-NBI (upper panel) and the histopathological papillary structure (lower panel). Adapted from Kanemitsu T, et al. Gastric Cancer 2014;17:469-477, with permission from Springer Nature. ${ }^{20}$
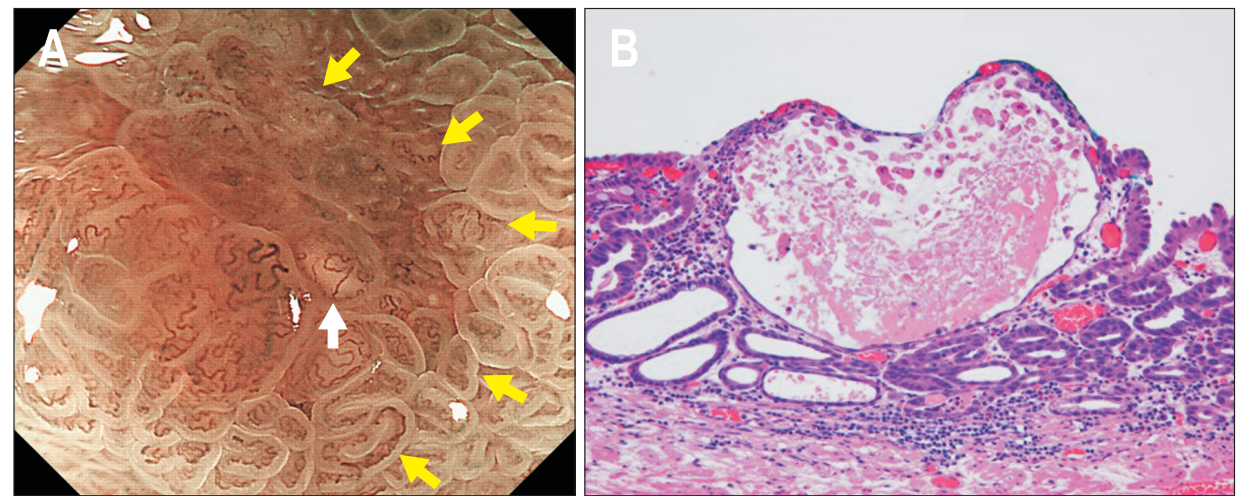

Fig. 7. Endoscopic image of the white globe appearance (WGA) and a corresponding histopathological image. (A) Representative endoscopic image of the WGA visualized using magnifying endoscopy with narrow-band imaging at maximal magnification (white arrow). Yellow arrows indicate a demarcation line. (B) Histological image of intraglandular necrotic debris (IND). By a careful histological investigation, this IND was proven to be identical to the WGA. Adapted from Doyama H, et al. Endosc Int Open 2015;3:E120-E124. ${ }^{21}$ 
a VEC pattern can be a useful indicator of high-grade of malignancy.

\section{White globe appearance}

White globe appearance (WGA) is defined as small (less than $1 \mathrm{~mm}$ ) white lesion with a globular shape located underneath the gastric epithelium in M-NBI observations (Fig. 7A). ${ }^{21}$ In detailed histological examinations, WGA corresponds to intraglandular necrotic debris, ${ }^{22}$ defined as eosinophilic material with necrotic epithelial fragments within the lumen of a dilated gland (Fig. 7B). ${ }^{21}$ The WGA prevalence in EGC, low-grade adenoma (LGA), and noncancerous lesions were $20 \%, 0 \%$, and $2.5 \%$, respectively. ${ }^{21,23}$ WGA is a finding specific to gastric cancer if found to exist inside the DL. In addition, the following features were obtained from previous clinical studies on WGA..$^{21,23,24}$ The presence of WGA means having a glandular structure. That is, the WGA is present in cancers with differenti- ated components and absent in pure undifferentiatedtype EGC. The WGA present in EGC is likely to occur frequently, mostly distributed near the margin (around just inside the DL), and is useful for delineation of the horizontal extent of EGC. Patients confirmed to have cancer with WGA tend to have multiple gastric cancers. The WGA is a finding that has a high interobserver agreement, and is an easily learnable marker for EGC, irrespective of endoscopists' experience. $^{24}$

\section{Multiple convex demarcation line}

The multiple convex demarcation line is a DL, which is protruded toward the inside, occasionally found in noncancerous depressed lesions (Fig. 8). ${ }^{25}$ The sensitivity, specificity, and positive predictive value of a multiple convex demarcation line for noncancerous lesions were $38 \%, 91 \%$, and $97 \%$, respectively. Analyzing the shape of DL is useful to distinguish between cancer and non-cancer.
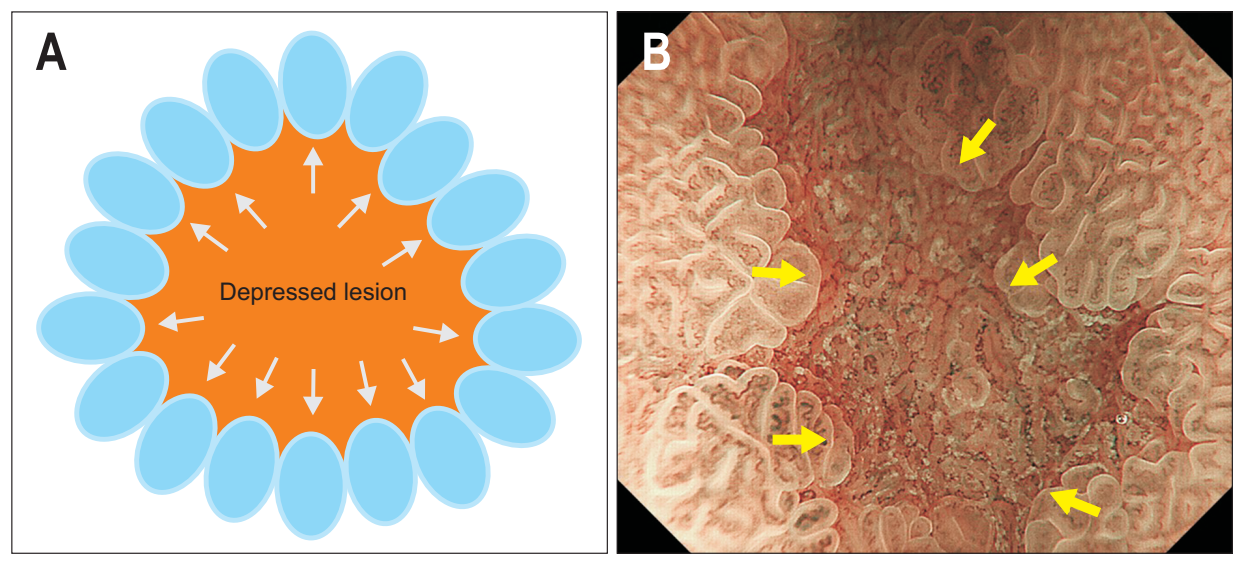

Fig. 8. Schematic diagram and an endoscopic image of a multiple convex demarcation line (MCDL). (A) Diagram of an MCDL. (B) Representative magnifying endoscopy with narrow-band imaging of the MCDL. The demarcation line (yellow arrows) forms multiple convex shapes on the inside. Adapted from Kanesaka T, et al. Endosc Int Open 2018;6:E145E155. ${ }^{25}$
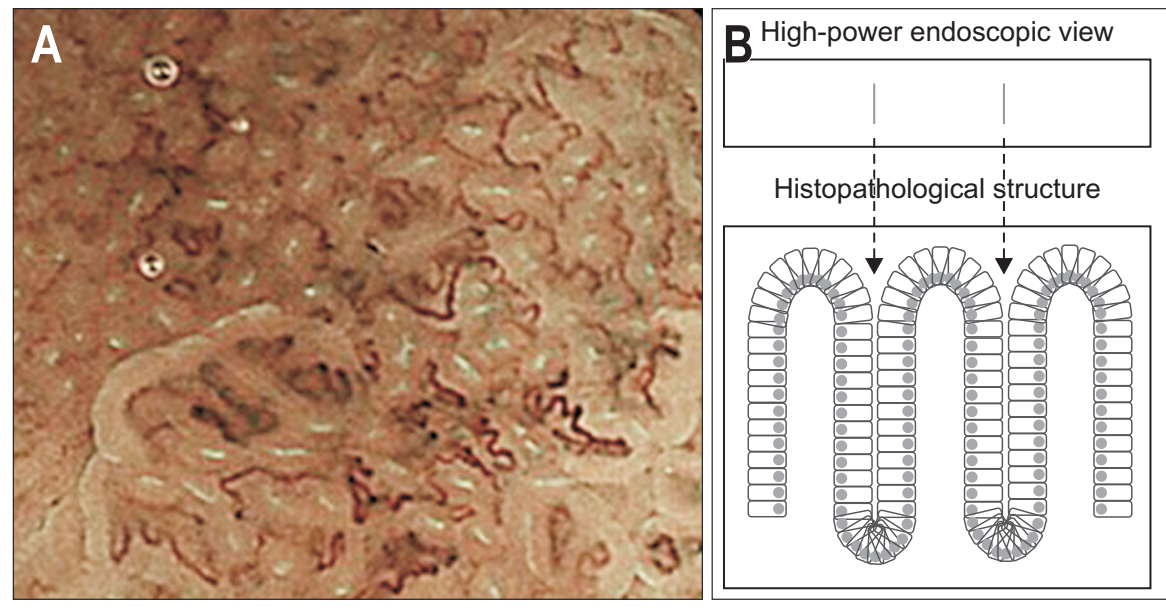

Histopathological structure
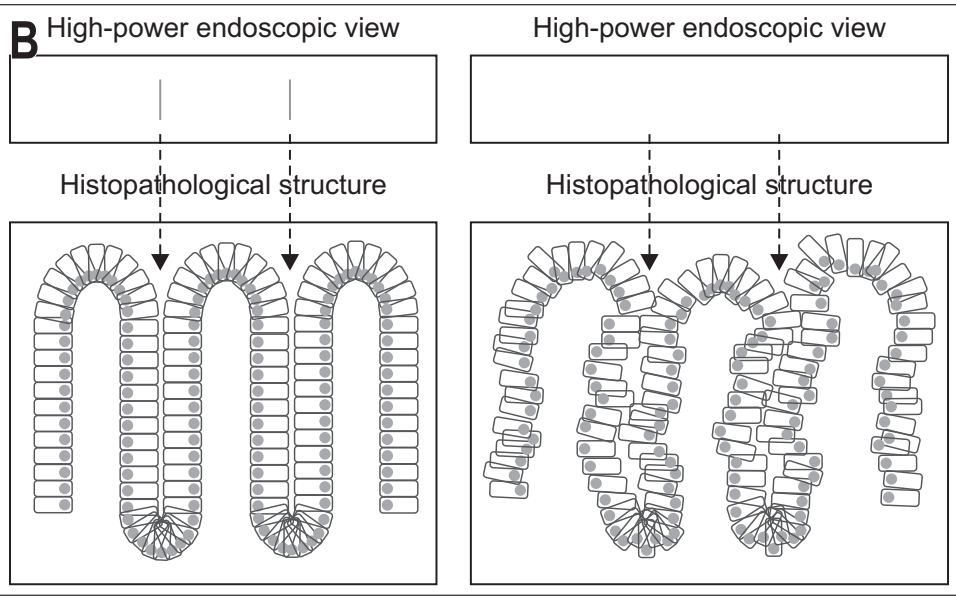

Fig. 9. Endoscopic image of dense-type crypt openings (COs) and a corresponding histopathological image. (A) Representative endoscopic image of dense-type $\mathrm{CO}$ s observed by magnifying endoscopy with narrow-band imaging with maximal magnification in low-grade adenoma. Numerous whitish, slit-like COs are observed. (B) COs are visualized as slit-like structures when histologically organized marginal crypt epithelium (MCE) are aligned in a regular manner (left image). On the other hand, $\mathrm{CO}$ s are not clearly visualized when MCE are aligned in an irregular manner (right image). Adapted from Kanesaka T, et al. Dig Endosc 2014;26:57-62. ${ }^{26}$ 


\section{Dense-type CO}

Slit-like CO is occasionally visualized in LGA by using M-NBI. Dense-type CO is defined as 20 or more slitlike COs recognized in one M-NBI image (Fig. 9). ${ }^{26}$ The sensitivity, specificity, and accuracy of dense-type CO for discriminating LGA from EGC were $90 \%, 88 \%$, and $88 \%$, respectively. Therefore, the dense-type $\mathrm{CO}$ is a useful indicator to discriminate LGA from EGC.

\section{Diagnosis of histological differentiation of EGC by M-NBI}

Subclassification of MV and MS patterns were proposed to predict histological patterns of gastric cancer. Absence of MS pattern (Fig. 5C) is defined as an area where neither MCE nor WOS is observed using M-NBI, indicating an undifferentiated-type EGC. ${ }^{27}$ In addition, the fine network pattern (Fig. 10A) was reported to be an MV pattern observed as a network-like mesh, indicating a differentiatedtype EGC. The corkscrew pattern (Fig. 10B) was reported to be an MV pattern observed as an isolated and disordered like corkscrew, indicating an undifferentiated-type EGC. ${ }^{28-30}$ Nevertheless, these findings have not been proven to be useful by well-designed prospective study. Accordingly, clinical application of these findings for predicting the histological differentiation is limited.

\section{MAGNIFYING ENDOSCOPY SIMPLE DIAGNOSTIC ALGORITHM FOR EARLY GASTRIC CANCER}

Many previous studies demonstrated that M-NBI is a highly accurate technique for diagnosing EGC. Based on these published data, the Japanese Gastroenterological Association (JGA), the Japan Gastroenterological Endoscopy Society (JGES), the Japanese Gastric Cancer Association (JGCA), and the World Endoscopy Organization (WEO) jointly proposed a unified algorithm for diagnosis of EGC using M-NBI (Fig. 11). ${ }^{15}$ The VS classification system constitutes the framework of this diagnostic algorithm. The diagnostic procedure along with magnifying endoscopy simple diagnostic algorithm for early gastric cancer (MESDA-G) is as follows. When a suspicious lesion is detected by C-WLI and switching to M-NBI, identification of a DL between the lesion and the background mucosa is the first step in distinguishing EGC from a noncancerous lesion.

If a DL is absent, the lesion is diagnosed as noncancerous. If a DL is identified, then MV and MS patterns within the DL should be determined. If either an irregular MV pattern or an irregular MS pattern is absent, the lesion is diagnosed as noncancerous. If an irregular MV pattern and/or an irregular MS pattern can be identified, the lesion is diagnosed as a cancerous lesion. However, the diagnosis of a signet-ring cell or undifferentiated-type carcinoma that shows a pale superficial depressed/flat lesion by CWLI, which is the limitation of VS classification system, is also the limitation in this algorithm. ${ }^{9}$

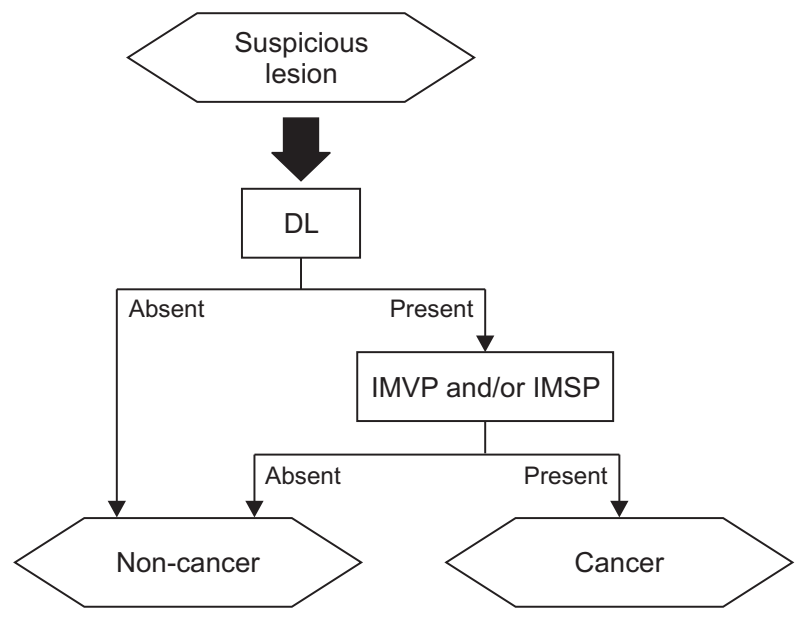

Fig. 11. Magnifying endoscopy simple diagnostic algorithm for early gastric cancer.

DL, demarcation line; IMVP, irregular microvascular pattern; IMSP, irregular microsurface pattern. Adapted from Muto M, et al. Dig Endosc 2016;28:379-393. ${ }^{15}$
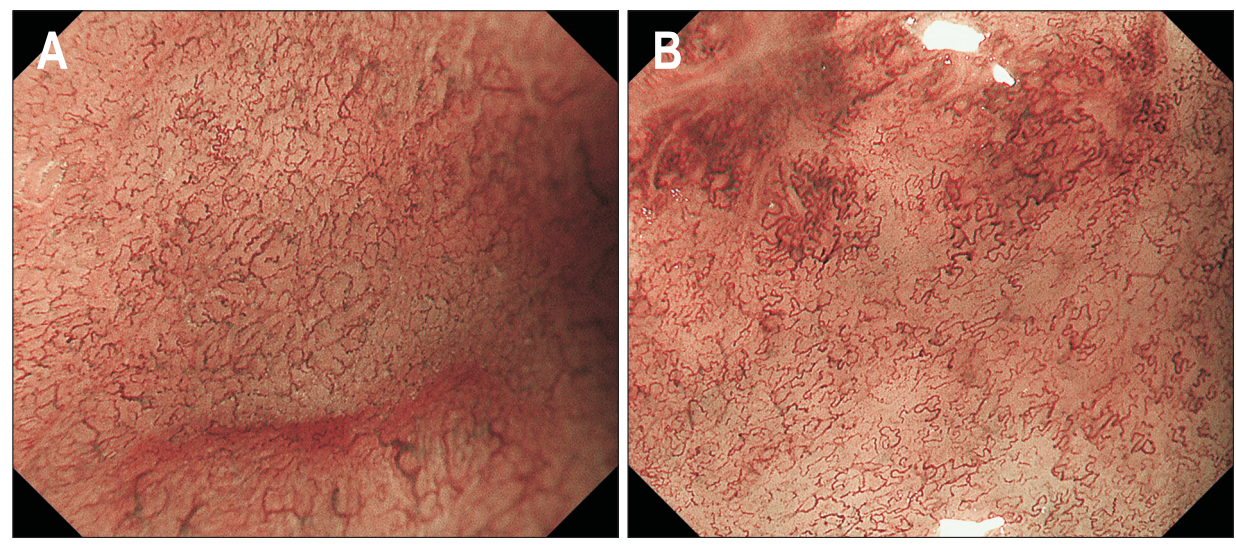

Fig. 10. Representative endoscopic image of the subclassification of irregular microvascular patterns visualized using magnifying endoscopy with narrow-band imaging with maximal magnification. (A) Fine network pattern and $(B)$ corkscrew pattern. 


\section{CONCLUSION AND FUTURE DIRECTION}

To demonstrate the basics for the diagnosis of EGC using magnifying endoscopy with image enhanced technique, we outlined the principles and clinical applications of the VS classification system and MESDA-G. Although $\mathrm{M}-\mathrm{NBI}$ is considered to have a huge contribution in clinical practice, acquiring skill at M-NBI diagnosis using the VS classification system requires considerable effort. To help deal with this challenge, we have developed an elearning system to teach endoscopic diagnosis of EGC using M-NBI based on the VS classification system, and evaluated the efficacy of the system by conducting a multicenter randomized controlled study in Japan, ${ }^{31,32}$ which proved that the e-learning system contributes to improving diagnostic accuracy. We are now working on developing an international version of the e-learning system to help more endoscopists learn gastric M-NBI diagnosis using the VS classification system. Some other important factors for improvement of diagnostic accuracy include imaging with the right focus at maximum magnification and using the right anatomical terminologies in diagnosis. Endoscopists are expected to pay careful attention to each and every case of endoscopic observations, with focus on improving their "technique" and "experience" as well as "knowledge."

\section{CONFLICTS OF INTEREST}

No potential conflict of interest relevant to this article was reported.

\section{ORCID}

Hisashi Doyama https://orcid.org/0000-0002-7457-0167 Hiroyoshi Nakanishi

https://orcid.org/0000-0003-0488-2082

Kenshi Yao

$$
\text { https://orcid.org/0000-0003-0863-3649 }
$$

\section{REFERENCES}

1. Tajiri H, Niwa H. Proposal for a consensus terminology in endoscopy: how should different endoscopic imaging techniques be grouped and defined? Endoscopy 2008;40:775778.

2. Yao K. Microanatomies as visualized using magnifying endoscopy with narrow bandimaging in the stomach: which microanatomical structures can we visualize in the glandular epithelium using narrow band imaging, and how is this achieved? In: Yao K, ed. Zoom gastroscopy. Tokyo: Springer, 2013:57-69.

3. Yao K. Clinical application of magnifying endoscopy with narrow-band imaging in the stomach. Clin Endosc 2015;48:481-490.

4. Yao K, Oishi T, Matsui T, Yao T, Iwashita A. Novel magnified endoscopic findings of microvascular architecture in intramucosal gastric cancer. Gastrointest Endosc 2002;56:279284.

5. Yao K, Iwashita A, Tanabe H, et al. Novel zoom endoscopy technique for diagnosis of small flat gastric cancer: a prospective, blind study. Clin Gastroenterol Hepatol 2007;5:869878.

6. Yao K, Anagnostopoulos GK, Ragunath K. Magnifying endoscopy for diagnosing and delineating early gastric cancer. Endoscopy 2009;41:462-467.

7. Ezoe Y, Muto M, Uedo N, et al. Magnifying narrowband imaging is more accurate than conventional white-light imaging in diagnosis of gastric mucosal cancer. Gastroenterology 2011;141:2017-2025.

8. Yamada S, Doyama H, Yao K, et al. An efficient diagnostic strategy for small, depressed early gastric cancer with magnifying narrow-band imaging: a post-hoc analysis of a prospective randomized controlled trial. Gastrointest Endosc 2014;79:55-63.

9. Yao K, Doyama H, Gotoda T, et al. Diagnostic performance and limitations of magnifying narrow-band imaging in screening endoscopy of early gastric cancer: a prospective multicenter feasibility study. Gastric Cancer 2014;17:669679.

10. Uedo N, Ishihara R, Iishi $\mathrm{H}$, et al. A new method of diagnosing gastric intestinal metaplasia: narrow-band imaging with magnifying endoscopy. Endoscopy 2006;38:819-824.

11. Yao K, Iwashita A, Tanabe H, et al. White opaque substance within superficial elevated gastric neoplasia as visualized by magnification endoscopy with narrow-band imaging: a new optical sign for differentiating between adenoma and carcinoma. Gastrointest Endosc 2008;68:574-580.

12. Yao K, Iwashita A, Nambu M, et al. Nature of white opaque substance in gastric epithelial neoplasia as visualized by magnifying endoscopy with narrow-band imaging. Dig Endosc 2012;24:419-425.

13. Ueo T, Yonemasu H, Yada N, et al. White opaque substance represents an intracytoplasmic accumulation of lipid droplets: immunohistochemical and immunoelectron microscopic investigation of 26 cases. Dig Endosc 2013;25:147155.

14. Gannon B, Browning J, O’Brien P, Rogers P. Mucosal microvascular architecture of the fundus and body of human stomach. Gastroenterology 1984;86(5 Pt 1):866-875.

15. Muto M, Yao K, Kaise M, et al. Magnifying endoscopy sim- 
ple diagnostic algorithm for early gastric cancer (MESDA-G). Dig Endosc 2016;28:379-393.

16. Yagi K, Nakamura A, Sekine A. Characteristic endoscopic and magnified endoscopic findings in the normal stomach without Helicobacter pylori infection. J Gastroenterol Hepatol 2002;17:39-45.

17. Nagahama T, Yao K, Maki S, et al. Usefulness of magnifying endoscopy with narrow-band imaging for determining the horizontal extent of early gastric cancer when there is an unclear margin by chromoendoscopy (with video). Gastrointest Endosc 2011;74:1259-1267.

18. Nagahama T, Yao K, Uedo N, et al. Delineation of the extent of early gastric cancer by magnifying narrow-band imaging and chromoendoscopy: a multicenter randomized controlled trial. Endoscopy 2018;50:566-576.

19. Kanesaka T, Uedo N, Yao K, et al. A significant feature of microvessels in magnifying narrow-band imaging for diagnosis of early gastric cancer. Endosc Int Open 2015;3:E590-E596.

20. Kanemitsu T, Yao K, Nagahama T, et al. The vessels within epithelial circle (VEC) pattern as visualized by magnifying endoscopy with narrow-band imaging (ME-NBI) is a useful marker for the diagnosis of papillary adenocarcinoma: a case-controlled study. Gastric Cancer 2014;17:469-477.

21. Doyama H, Yoshida N, Tsuyama S, et al. The "white globe appearance" (WGA): a novel marker for a correct diagnosis of early gastric cancer by magnifying endoscopy with narrow-band imaging (M-NBI). Endosc Int Open 2015;3:E120E124.

22. Watanabe Y, Shimizu M, Itoh T, Nagashima K. Intraglandular necrotic debris in gastric biopsy and surgical specimens. Ann Diagn Pathol 2001;5:141-147.

23. Yoshida N, Doyama H, Nakanishi H, et al. White globe appearance is a novel specific endoscopic marker for gastric cancer: a prospective study. Dig Endosc 2016;28:59-66.

24. Omura H, Yoshida N, Hayashi T, et al. Interobserver agreement in detection of "white globe appearance" and the abil- ity of educational lectures to improve the diagnosis of gastric lesions. Gastric Cancer 2017;20:620-628.

25. Kanesaka T, Uedo N, Yao K, et al. Multiple convex demarcation line for prediction of benign depressed gastric lesions in magnifying narrow-band imaging. Endosc Int Open 2018;6:E145-E155

26. Kanesaka T, Sekikawa A, Tsumura T, et al. Dense-type crypt opening seen on magnifying endoscopy with narrowband imaging is a feature of gastric adenoma. Dig Endosc 2014;26:57-62.

27. Kanesaka T, Sekikawa A, Tsumura T, et al. Absent microsurface pattern is characteristic of early gastric cancer of undifferentiated type: magnifying endoscopy with narrow-band imaging. Gastrointest Endosc 2014;80:1194-1198.

28. Nakayoshi T, Tajiri H, Matsuda K, Kaise M, Ikegami M, Sasaki H. Magnifying endoscopy combined with narrow band imaging system for early gastric cancer: correlation of vascular pattern with histopathology (including video). Endoscopy 2004;36:1080-1084.

29. Ok KS, Kim GH, Park do Y, et al. Magnifying endoscopy with narrow band imaging of early gastric cancer: correlation with histopathology and mucin phenotype. Gut Liver 2016;10:532-541.

30. Horiuchi Y, Tokai Y, Yamamoto N, et al. Additive effect of magnifying endoscopy with narrow-band imaging for diagnosing mixed-type early gastric cancers. Dig Dis Sci 2020;65:591-599.

31. Nakanishi $H$, Doyama $H$, Ishikawa $H$, et al. Evaluation of an e-learning system for diagnosis of gastric lesions using magnifying narrow-band imaging: a multicenter randomized controlled study. Endoscopy 2017;49:957-967.

32. Ikehara H, Doyama H, Nakanishi H, et al. Analysis of factors related to poor outcome after e-learning training in endoscopic diagnosis of early gastric cancer using magnifying narrow-band imaging. Gastrointest Endosc 2019;90:440447. 